(C) 2003 American Chemical Society, J. Org. Chem., Kamimura jo030052h Supporting Info Page 1

\title{
Convenient Synthesis of 2-Benzazepines via Radical Cyclization
}

Akio Kamimura, ${ }^{*, a}$ Yohei Taguchi, ${ }^{b}$ Yoji Omata ${ }^{a}$ and Masahiko Hagihara ${ }^{b}$

${ }^{a}$ Department of Applied Chemistry, Faculty of Engineering, Yamaguchi University, Ube 755-8611 Japan

${ }^{b}$ Ube Laboratory, Ube Industries Ltd., Ube 755-8633 Japan

\section{Supporting Information}

Table of contents

Experimental section for the preparation of compounds 2 and 3

\section{Experimental}

\section{General}

All ${ }^{1} \mathrm{H}$ and ${ }^{13} \mathrm{C}$ NMR spectra were measured in $\mathrm{CDCl}_{3}$ or DMSO-d6 and recorded on JEOL EX-270 (270 MHz for $\mathrm{H}$ and $67.5 \mathrm{MHz}$ for $\mathrm{C})$ spectrometer. All the reactions were performed under nitrogen atmosphere unless otherwise mentioned. Solvents used in the reaction described here were dried over appropriate drying agents ( $\mathrm{K}$ for THF, Na for ether, benzene and toluene, and $\mathrm{CaH}_{2}$ for all other solvents) and distilled under nitrogen before use. $\mathrm{Bu}_{3} \mathrm{SnH}$ was purchased from $\mathrm{TCI}$, which were used without further purification.

\section{Preparation of N-(2-Bromo-5-methoxybenzyl)-2,2,2-trifluoroethylamine [2a].}

General Procedure: To a mixture of 2-bromo-5-methoxybenzaldehyde (3.26 g, 15.2 $\mathrm{mmol})$ and 2,2,2-trofluoroethylamine $(1.40 \mathrm{~mL}, 17.6 \mathrm{mmol})$ in methanol $(20 \mathrm{~mL})$ at $0^{\circ} \mathrm{C}$ was added $\mathrm{NaBH}_{3} \mathrm{CN}(1.70 \mathrm{~g}, 27.1 \mathrm{mmol})$ and acetic acid $(1.00 \mathrm{~mL}, 18.9 \mathrm{mmol})$. The 
(C) 2003 American Chemical Society, J. Org. Chem., Kamimura jo030052h Supporting Info Page 2

reaction mixture was allowed to stir at ambient temperature for $7 \mathrm{~h}$. After removal of methanol in vacuo, water $(50 \mathrm{~mL})$ was added to the residue and the resulting biphasic mixture was extracted with ether $(3 \times 30 \mathrm{~mL})$. Combined organic phase was dried over $\mathrm{Na}_{2} \mathrm{SO}_{4}$. After filtration and evaporation of organic solvent, the crude product was purified through flash chromatography (hexane then hexane-ethyl acetate) to give $2 \mathbf{a}$ in $87 \%$ yield $(3.94 \mathrm{~g})$. Colorless oil. ${ }^{1} \mathrm{H}$ NMR $\left(\mathrm{CDCl}_{3}\right) \delta 1.87(\mathrm{~s}, 1 \mathrm{H}), 3.18(\mathrm{q}, 2 \mathrm{H}, J=9.4$ $\mathrm{Hz}), 3.79(\mathrm{~s}, 3 \mathrm{H}), 3.93(\mathrm{~s}, 2 \mathrm{H}), 6.70(\mathrm{dd}, 1 \mathrm{H}, J=3.3,8.6 \mathrm{~Hz}), 6.97(\mathrm{~d}, 1 \mathrm{H}, J=3.0 \mathrm{~Hz}$ ), $7.43(\mathrm{~d}, 1 \mathrm{H}, J=8.9 \mathrm{~Hz}) .{ }^{13} \mathrm{C}-\mathrm{NMR}\left(\mathrm{CDCl}_{3}\right) \delta 49.5(\mathrm{q}, J=31.8 \mathrm{~Hz}), 52.9,55.3,113.8$, $114.4,115.7,125.5(\mathrm{q}, J=279 \mathrm{~Hz}), 133.4,139.0,159.0$.

Other amines 2 were prepared in a similar manner.

N-Benzyl (2-bromo-5-methoxybenzyl)amine [2b]: ${ }^{1} \mathrm{H}-\mathrm{NMR}\left(\mathrm{CDCl}_{3}\right) \delta 2.04(\mathrm{~s}, 1 \mathrm{H})$, $3.79(\mathrm{~s}, 3 \mathrm{H}), 3.81(\mathrm{~s}, 2 \mathrm{H}), 3.84(\mathrm{~s}, 2 \mathrm{H}), 6.69(\mathrm{dd}, 1 \mathrm{H}, J=3.0,8.6 \mathrm{~Hz}), 6.99$ (d, $1 \mathrm{H}, \mathrm{J}$ $=3.0 \mathrm{~Hz}), 7.27-7.44(\mathrm{~m}, 6 \mathrm{H}) .{ }^{13} \mathrm{C}-\mathrm{NMR}\left(\mathrm{CDCl}_{3}\right) \delta 52.9,53.0,55.3,114.1,115.7,126.9$, $128.1,128.3,133.1,139.9,140.0,158.9$.

N-(2-Bromo-5-methoxybenzyl)-butylamine [2c]: ${ }^{1} \mathrm{H}-\mathrm{NMR}\left(\mathrm{CDCl}_{3}\right) \delta 0.92(\mathrm{t}, 3 \mathrm{H}, J=$ $7.3 \mathrm{~Hz}), 1.32-1.41(\mathrm{~m}, 2 \mathrm{H}), 1.46-1.55(\mathrm{~m}, 2 \mathrm{H}), 1.61(\mathrm{~s}, 1 \mathrm{H}), 2.63(\mathrm{t}, 2 \mathrm{H}, J=7.1 \mathrm{~Hz})$, $3.79(\mathrm{~s}, 3 \mathrm{H}), 3.81(\mathrm{~s}, 2 \mathrm{H}), 6.67(\mathrm{dd}, 1 \mathrm{H}, J=3.3,8.6 \mathrm{~Hz}), 6.97(\mathrm{~d}, 1 \mathrm{H}, J=3.0 \mathrm{~Hz})$, $7.41(\mathrm{~d}, 1 \mathrm{H}, J=8.9 \mathrm{~Hz}) .{ }^{13} \mathrm{C}-\mathrm{NMR}\left(\mathrm{CDCl}_{3}\right) \delta 13.9,20.3,32.1,48.8,53.8,55.2,113.9$, $114.0,115.6,133.0,140.3,158.8$.

N-(2-Bromobenzyl)-2,2,2-trifluoroethylamine [2d]: ${ }^{1} \mathrm{H}-\mathrm{NMR}\left(\mathrm{CDCl}_{3}\right) \delta 1.83(\mathrm{br}, 1$ $\mathrm{H}), 3.18(\mathrm{q}, 2 \mathrm{H}, J=9.2 \mathrm{~Hz}), 3.98(\mathrm{~s}, 2 \mathrm{H}), 7.12-7.58(\mathrm{~m}, 4 \mathrm{H}) .{ }^{13} \mathrm{C}-\mathrm{NMR}\left(\mathrm{CDCl}_{3}\right) \delta 49.4$ $(\mathrm{q}, J=31.7 \mathrm{~Hz}), 52.9,123.8,125.5(\mathrm{q}, J=279.5 \mathrm{~Hz}), 127.5,128.9,130.1,132.9,138.0$.

\section{Preparation of}

$N$-(2-Bromo-5-methoxybenzyl)2-methylene- $N$-(2,2,2-trifluoroethyl)-succinylamide4-ethyl ester [3a]. General Procedure: To a solution of $2 \mathrm{a}(2.88 \mathrm{~g}, 17.7 \mathrm{mmol}), \mathrm{Et}_{3} \mathrm{~N}$ 
(C) 2003 American Chemical Society, J. Org. Chem., Kamimura jo030052h Supporting Info Page 3

(2.50 $\mathrm{mL}, 17.7 \mathrm{mmol})$ and $\operatorname{DMAP}(0.1 \mathrm{~g})$ in $\mathrm{CH}_{2} \mathrm{Cl}_{2}(25 \mathrm{~mL})$ was added (monoethyl)itaconoyl choloride, prepared from monoethyl itaconate $(2.80 \mathrm{~g}, 17.7$ $\mathrm{mmol})$ and $\mathrm{SOCl}_{2}(1.48 \mathrm{~mL}, 20.4 \mathrm{mmol})$, at $-50^{\circ} \mathrm{C}$. The reaction mixture was allowed to warm to room temperature for $12 \mathrm{~h}$. $\mathrm{HClaq}(1 \mathrm{M}, 30 \mathrm{~mL})$ was added to the reaction mixture and the resulting solution was extracted with $\mathrm{CH}_{2} \mathrm{Cl}_{2}(3 \times 40 \mathrm{~mL})$. The organic phase was combined, washed with $\mathrm{NaHCO}_{3}(50 \mathrm{~mL})$ and dried over $\mathrm{Na}_{2} \mathrm{SO}_{4}$. After filtration and evaporation of organic solvent, the crude product was purified through flash chromatography (hexane then hexane-ethyl acetate) to give $3 \mathrm{a}$ in $77 \%$ yield (3.26 g). Colorless oil. ${ }^{1} \mathrm{H} \mathrm{NMR}\left(\mathrm{CDCl}_{3}\right) \delta 1.26(\mathrm{t}, 3 \mathrm{H}, J=7.1 \mathrm{~Hz}), 3.49(\mathrm{~s}, 2 \mathrm{H}), 3.77(\mathrm{~s}, 3$ H), $4.14(\mathrm{q}, 2 \mathrm{H}, J=7.2 \mathrm{~Hz}) ; 4.09-4.18(\mathrm{~m}, 2 \mathrm{H}), 4.91(\mathrm{br}, 2 \mathrm{H}), 5.45(\mathrm{~m}, 2 \mathrm{H}), 6.72(\mathrm{~d}$, $1 \mathrm{H}, J=8.6 \mathrm{~Hz}), 6.80(\mathrm{~d}, 1 \mathrm{H}, \mathrm{J}=2.6 \mathrm{~Hz}), 7.46(\mathrm{~d}, 1 \mathrm{H}, J=8.6 \mathrm{~Hz}) .{ }^{13} \mathrm{C}-\mathrm{NMR}\left(\mathrm{CDCl}_{3}\right)$ $\delta 14.0,39.9,48.8,53.2,55.4,61.1,113.2,114.0,114.6,120.2,124.3$ (q, $J=280.8 \mathrm{~Hz})$, $133.5,135.6,135.8,159.4,170.4,172.3$.

Other amides 3 were prepared in a similar manner.

$N$-(2-Bromo-5-methoxybenzyl)-2-methyl- $N$-(2,2,2-trifluoroethyl)acrylamide [3b]: ${ }^{1} \mathrm{H}-\mathrm{NMR}\left(\mathrm{CDCl}_{3}\right) \delta 2.00(\mathrm{~s}, 3 \mathrm{H}), 3.78(\mathrm{~s}, 3 \mathrm{H}), 4.02(\mathrm{q}, 2 \mathrm{H}, J=8.9 \mathrm{~Hz}), 4.80(\mathrm{~s}, 2 \mathrm{H})$, $5.16(\mathrm{~s}, 1 \mathrm{H}), 5.29(\mathrm{~s}, 1 \mathrm{H}), 6.70-6.77(\mathrm{~m}, 1 \mathrm{H}), 6.74(\mathrm{dd}, 1 \mathrm{H}, J=3.0,8.9 \mathrm{~Hz}), 7.47(\mathrm{~d}, 1$ $\mathrm{H}, J=8.9 \mathrm{~Hz}) .{ }^{13} \mathrm{C}-\mathrm{NMR}\left(\mathrm{CDCl}_{3}\right) \delta 20.3,48.3,52.6,55.4,113.4,114.7,114.9,116.9$, $124.3(\mathrm{q}, J=280 \mathrm{~Hz}), 134.0,135.8,139.3,159.3,173.6$. Anal. calc. for $\mathrm{C}_{14} \mathrm{H}_{15} \mathrm{BrF}_{3} \mathrm{NO}_{2}$ : C, 45.92; H, 4.13; N, 3.83. Found: C, 45.64; H, 4.00; N, 3.71 .

$N$-(2-Bromo-5-methoxybenzyl)-N-(2,2,2-trifluoroethyl)acrylamide [3c]: ' $\mathrm{H}-\mathrm{NMR}$ (DMSO-d6) $\delta 3.43$ (s, $2 \mathrm{H}$ ), 3.80 (s, $3 \mathrm{H}$ ), $4.43(\mathrm{q}, 1 \mathrm{H}, J=9.6 \mathrm{~Hz}$ ), 4.62 (q, $1 \mathrm{H}, J=8.9$ $\mathrm{Hz}), 4.71(\mathrm{~s}, 1 \mathrm{H}), 4.86(\mathrm{~s}, 1 \mathrm{H}), 5.87$ (dd, $1 \mathrm{H}, J=10.7,24.4 \mathrm{~Hz}), 6.36(\mathrm{dd}, 1 \mathrm{H}, J=2.3$, $16.5 \mathrm{~Hz}), 6.57-6.65(\mathrm{~m}, 2 \mathrm{H}), 6.92-7.10(\mathrm{~m}, 1 \mathrm{H}), 7.63(\mathrm{t}, 1 \mathrm{H}, J=8.9 \mathrm{~Hz}) .{ }^{13} \mathrm{C}-\mathrm{NMR}$ $\left(\mathrm{CDCl}_{3}\right) \delta 46.7(\mathrm{q}, J=32.0 \mathrm{~Hz}), 52.5,55.4,112.3,113.6,114.4,126.2,128.2(\mathrm{q}, \mathrm{J}=$ $272.8 \mathrm{~Hz}), 130.7,133.9,135.7,159.5,167.6$. Anal. calc. for $\mathrm{C}_{13} \mathrm{H}_{13} \mathrm{BrF}_{3} \mathrm{NO}_{2}$ : C, 44.34; 
(C) 2003 American Chemical Society, J. Org. Chem., Kamimura jo030052h Supporting Info Page 4

H, 3.72; N, 3.98. Found: C, 44.08; H, 3.81; N, 3.99

$N$-(2-Bromo-5-methoxybenzyl)2-methylene- $N$-benzylsuccinylamide-4-methyl ester

[3d]: ${ }^{1} \mathrm{H}-\mathrm{NMR}\left(\mathrm{CDCl}_{3}\right) \delta 3.54(\mathrm{~s}, 2 \mathrm{H}), 3.68(\mathrm{~m}, 3 \mathrm{H}), 3.78(\mathrm{~s}, 3 \mathrm{H}), 4.68-80(\mathrm{~m}, 4 \mathrm{H})$, 5.29-5.39 (m, $2 \mathrm{H}), 6.70(\mathrm{br}, 1 \mathrm{H}), 6.88(\mathrm{~d}, 1 \mathrm{H}, J=3.0 \mathrm{~Hz}), 7.22-7.65(\mathrm{~m}, 6 \mathrm{H})$.

${ }^{13} \mathrm{C}-\mathrm{NMR}\left(\mathrm{CDCl}_{3}\right) \delta 40.1,48.1,52.0,55.5,113.8,114.2,114.5,118.7,126.6,127.4$, $128.3,128.7,133.3,133.7,136.5,159.4,171.7(2 \mathrm{C})$. Anal. calc. for $\mathrm{C}_{21} \mathrm{H}_{22} \mathrm{BrNO}_{4}: \mathrm{C}$, $58.34 ; \mathrm{H}, 5.13 ; \mathrm{N}, 3.24$. Found: C, 58.09; H, 5.07; N, 3.28.

$N$-(2-Bromo-5-methoxybenzyl)-2-methyl- $N$-benzylacrylamide [3e]: ${ }^{1} \mathrm{H}-\mathrm{NMR}$ (DMSO-d6) $\delta 1.91$ (s, 3 H), $3.72(\mathrm{~s}, 3 \mathrm{H}), 4.47(\mathrm{br}, 2 \mathrm{H}), 4.58(\mathrm{br}, 2 \mathrm{H}), 5.08(\mathrm{br}, 1 \mathrm{H})$, $5.21(\mathrm{br}, 1 \mathrm{H}), 6.65(\mathrm{~d}, 1 \mathrm{H}, J=3.0 \mathrm{~Hz}), 6.85(\mathrm{dd}, 1 \mathrm{H}, J=3.0,8.6 \mathrm{~Hz}), 7.19-7.38(\mathrm{~m}, 5$

H), $7.50\left(\mathrm{~d}, 1 \mathrm{H}, J=8.6 \mathrm{~Hz}\right.$ ). ${ }^{13} \mathrm{C}-\mathrm{NMR}$ (DMSO-d6) $\delta 20.3,51.2(\mathrm{~m}), 55.3,113.9,112.0$, $114.6,115.1,127.3,128.6,133.4,136.6,136.9,140.0,158.9,172.2$. Anal. calc. for $\mathrm{C}_{19} \mathrm{H}_{20} \mathrm{BrNO}_{2}: \mathrm{C}, 60.97 ; \mathrm{H}, 5.39 ; \mathrm{N}, 3.74$. Found: $\mathrm{C}, 60.70 ; \mathrm{H}, 5.28 ; \mathrm{N}, 3.68$.

$N$-(2-Bromo-5-methoxybenzyl)2-methylene- $N$-butylsuccinylamide-4-methyl ester [3f]: ${ }^{1} \mathrm{H}-\mathrm{NMR}$ (DMSO-d6, $\left.80^{\circ} \mathrm{C}\right) \delta 0.93(\mathrm{t}, 3 \mathrm{H}, J=7.3 \mathrm{~Hz}), 1.33(\mathrm{~m}, 2 \mathrm{H}), 1.60(\mathrm{~m}, 2$ H), 3.38-3.43 (m, $2 \mathrm{H}), 3.46(\mathrm{~s}, 2 \mathrm{H}), 3.69(\mathrm{~s}, 3 \mathrm{H}), 3.84(\mathrm{~s}, 3 \mathrm{H}), 4.68(\mathrm{~s}, 2 \mathrm{H}), 5.35(\mathrm{~s}, 1$ H), $5.50(\mathrm{~s}, 1 \mathrm{H}), 6.87-6.93(\mathrm{~m}, 3 \mathrm{H}), 7.59(\mathrm{~d}, 2 \mathrm{H}, J=8.6 \mathrm{~Hz}) .{ }^{13} \mathrm{C}-\mathrm{NMR}\left(\mathrm{CDCl}_{3}\right) \delta$ $13.6,19.9,28.6$ and 30.5 (rotamer, $1 \mathrm{C}$ ), $39.7,45.5$ and 47.3 (rotamer, $1 \mathrm{C}$ ), 47.8 and 52.4 (rotamer, 1 C), 51.6, 55.3, 112.6, 113.8, 114.3, 118.0, 133.0, 133.3, 137.0, 159.2, 171.0, 171.1. Anal. calc. for $\mathrm{C}_{18} \mathrm{H}_{24} \mathrm{BrNO}_{4}$ : C, 54.28; $\mathrm{H}, 6.07 ; \mathrm{N}, 3.52$. Found: $\mathrm{C}, 54.58$; $H, 6.06 ; \mathrm{N}, 3.46$.

$N$-(2-Bromobenzyl)2-methylene- $N$-(2,2,2-trifluoroethyl)-succinylamide-4-methyl ester [3g]: ${ }^{1} \mathrm{H}-\mathrm{NMR}$ (DMSO-d6, $\left.60^{\circ} \mathrm{C}\right) \delta 3.37(\mathrm{~s}, 2 \mathrm{H}), 3.60(\mathrm{~s}, 3 \mathrm{H}), 4.26(\mathrm{q}, 2 \mathrm{H}, J=$ $9.2 \mathrm{~Hz}), 4.78(\mathrm{~s}, 2 \mathrm{H}), 5.33(\mathrm{~s}, 1 \mathrm{H}), 5.51(\mathrm{~s}, 1 \mathrm{H}), 7.21-7.28(\mathrm{~m}, 2 \mathrm{H}), 7.42(\mathrm{t}, 1 \mathrm{H}, J=$ $7.3 \mathrm{~Hz}), 7.64(\mathrm{~d}, 1 \mathrm{H}, J=8.0 \mathrm{~Hz}) .{ }^{13} \mathrm{C}-\mathrm{NMR}\left(\mathrm{CDCl}_{3}\right) \delta 39.7,45.1(\mathrm{~m}), 50.8(\mathrm{~m}), 52.1$, $120.2,123.1,124.3(\mathrm{q}, J=280.9 \mathrm{~Hz}), 128.0,128.6,133.3,134.7,135.8,171.0,172.2$. Anal. calc. for $\mathrm{C}_{15} \mathrm{H}_{15} \mathrm{BrF}_{3} \mathrm{NO}_{3}: \mathrm{C}, 45.70 ; \mathrm{H}, 3.84 ; \mathrm{N}, 3.55$. Found: $\mathrm{C}, 45.77 ; \mathrm{H}, 3.85 ; \mathrm{N}$, 
(C) 2003 American Chemical Society, J. Org. Chem., Kamimura jo030052h Supporting Info Page 5

3.49 .

$N$-(2-Bromobenzyl)-2-methyl- $N$-(2,2,2-trifluoroethyl)acrylamide [3h]: ${ }^{1} \mathrm{H}-\mathrm{NMR}$ $\left(\mathrm{CDCl}_{3}\right) \delta 1.98(\mathrm{~s}, 3 \mathrm{H}), 4.01(\mathrm{q}, 2 \mathrm{H}, J=8.9 \mathrm{~Hz}), 4.85(\mathrm{~s}, 2 \mathrm{H}), 5.15(\mathrm{~s}, 1 \mathrm{H}), 5.28(\mathrm{~s}, 1$ $\mathrm{H}), 7.17-7.36(\mathrm{~m}, 3 \mathrm{H}), 7.60(\mathrm{dd}, 1 \mathrm{H}, J=1.0,7.9 \mathrm{~Hz}) .{ }^{13} \mathrm{C}-\mathrm{NMR}\left(\mathrm{CDCl}_{3}\right) \delta 20.2,44.7$ (m), $52.9(\mathrm{~m}), 116.8,124.3(\mathrm{q}, J=282.0 \mathrm{~Hz}), 127.8,129.5,133.4,134.7,139.3,173.6$. Anal. calc. for $\mathrm{C}_{13} \mathrm{H}_{13} \mathrm{BrF}_{3} \mathrm{NO}$ : $\mathrm{C}, 46.45 ; \mathrm{H}, 3.90 ; \mathrm{N}, 4.17$. Found: $\mathrm{C}, 46.71 ; \mathrm{H}, 4.00 ; \mathrm{N}$, 4.12.

$N$-(2-Bromobenzyl)- $N$-(2,2,2-trifluoroethyl)acrylamide [3i]: ${ }^{1} \mathrm{H}-\mathrm{NMR}$ (DMSO-d6) $\delta$ $4.34(\mathrm{q}, 1 \mathrm{H}, J=9.4 \mathrm{~Hz}), 4.53(\mathrm{q}, 1 \mathrm{H}, J=8.9 \mathrm{~Hz}), 4.69(\mathrm{~s}, 1 \mathrm{H}), 4.84(\mathrm{~s}, 1 \mathrm{H}), 5.73(\mathrm{~d}$, $1 \mathrm{H}$ for rotamer A, $J=9.2 \mathrm{~Hz}$ ), $5.83(\mathrm{~d}, 1 \mathrm{H}$ for rotamer $\mathrm{B}, J=9.2 \mathrm{~Hz}), 6.27(\mathrm{dd}, 1 \mathrm{H}, \mathrm{J}$ $=2.3,16.5 \mathrm{~Hz}$ ), $6.55(\mathrm{dd}, 1 \mathrm{H}, \mathrm{J}=10.2,16.5 \mathrm{~Hz}), 6.93-7.68(\mathrm{~m}, 4 \mathrm{H}) .{ }^{13} \mathrm{C}-\mathrm{NMR}$ $\left(\mathrm{CDCl}_{3}\right) \delta 46.4$ (q, $\left.J=34.2 \mathrm{~Hz}\right), 52.4,126.2,127.8,128.4$ (q, $\left.J=276.3 \mathrm{~Hz}\right), 129.4$, 130.5, 133.2, 167.5. Anal. calc. for $\mathrm{C}_{12} \mathrm{H}_{11} \mathrm{BrF}_{3} \mathrm{NO}$ : $\mathrm{C}, 44.74 ; \mathrm{H}, 3.44 ; \mathrm{N}, 4.35$. Found: $\mathrm{C}$, $44.69 ; \mathrm{H}, 3.47 ; \mathrm{N}, 4.29$. 
(C) 2003 American Chemical Society, J. Org. Chem., Kamimura jo030052h Supporting Info Page 6

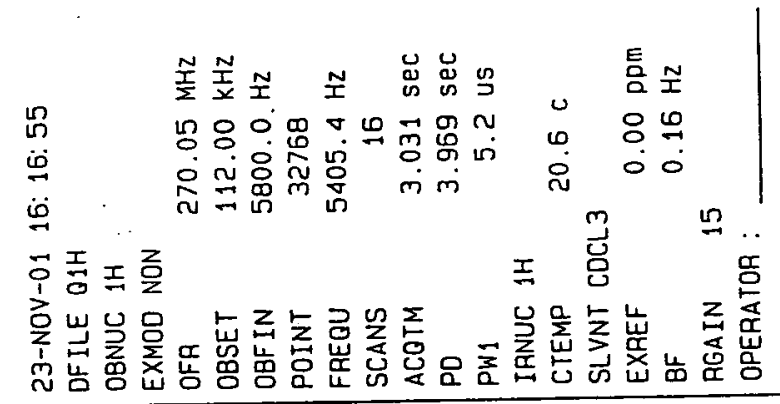

$000^{\circ} \longrightarrow$

$\angle D D^{\circ} 6 \longrightarrow$

$620.0 \longrightarrow$
$080.0 \longrightarrow$

$80 L^{\circ} \mathrm{BI} \longrightarrow$

29L'90 $\longrightarrow$

$\mathrm{S20} 0 \longrightarrow$

SSE. $82 \longrightarrow$

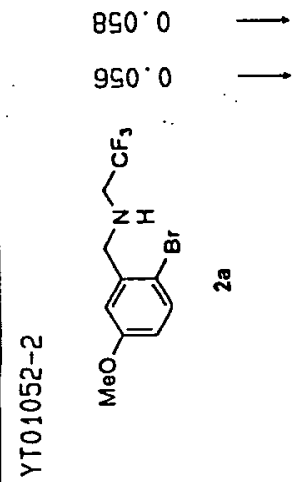


(ْ) 2003 American Chemical Society, J. Org. Chem., Kamimura jo030052h Supporting Info Page 7,
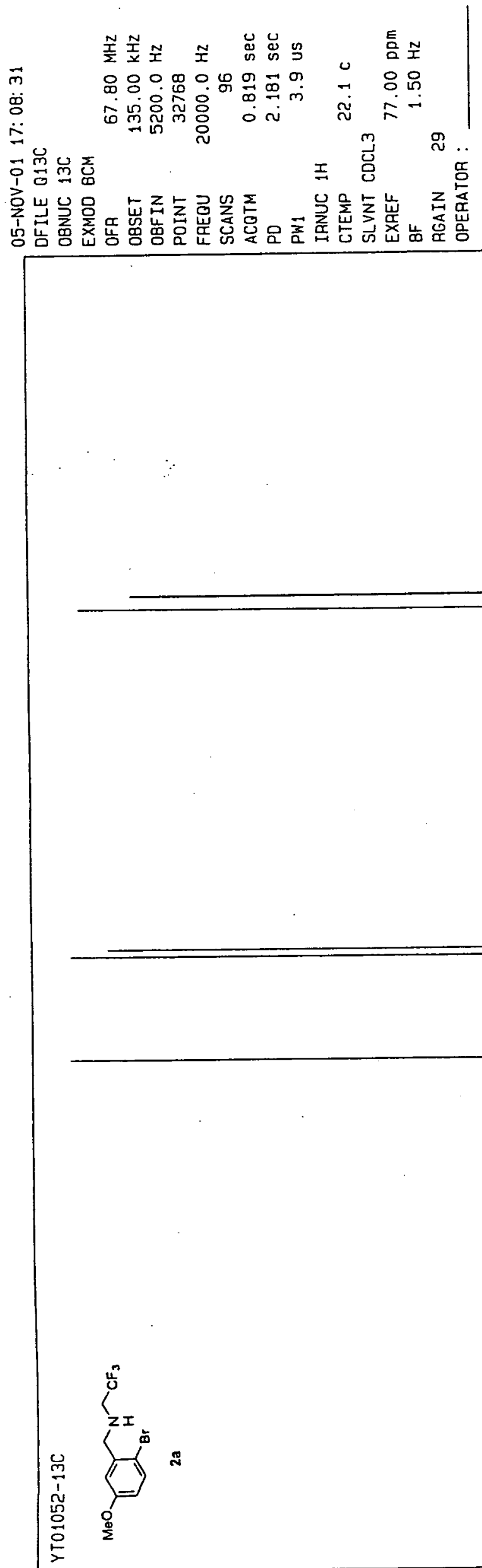
(c) 2003 American Chemical Society, J. Org. Chem., Kamimura jo030052h Supporting Info Page 8
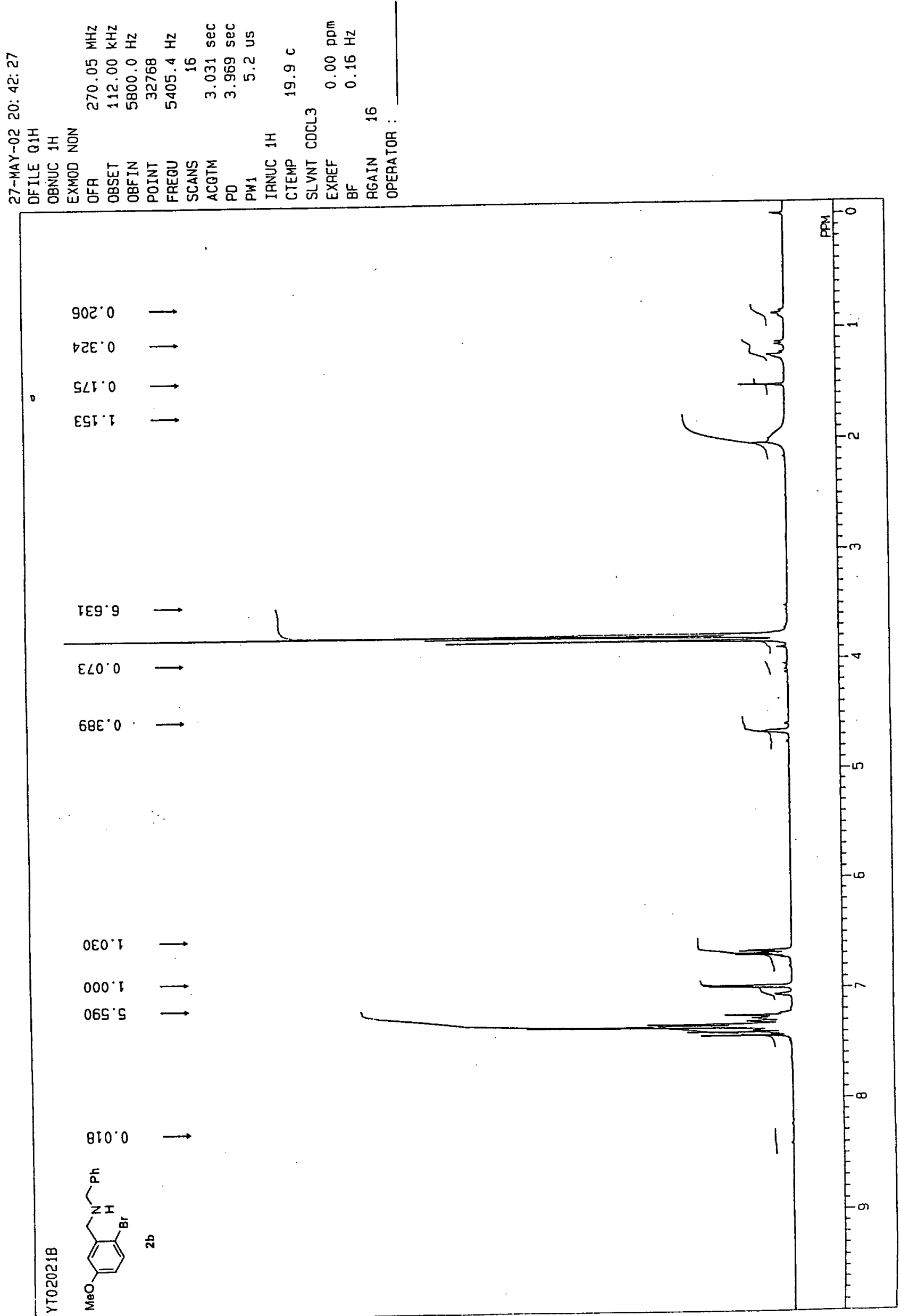
(C) 2003 Ämerican Chemical Society, J. Org. Chem., Kamimura jo030052h Supporting Info Page 9

: $\quad$ s9

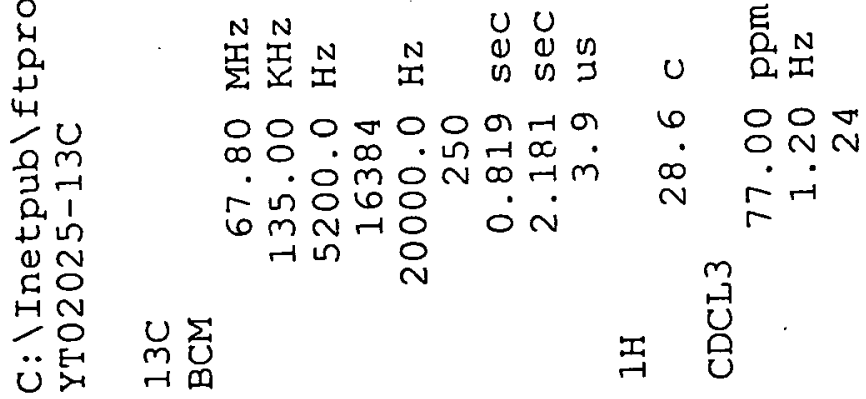

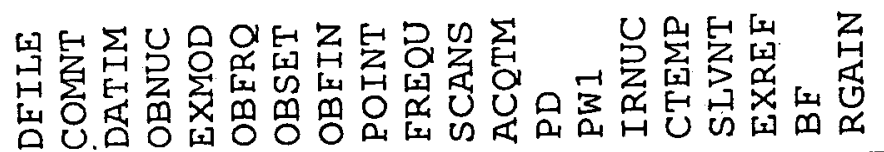

ช0

$\left.\begin{array}{l}I Z 6^{\circ} Z S \\ L D 0^{\circ} E S \\ S L Z \cdot S S\end{array}\right]$

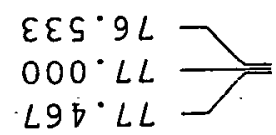

ZSO००IT—

TBL・STI-

$288 \cdot 92 \tau$

$950^{\circ} 8 Z T$

$8 T I \cdot \varepsilon \varepsilon \tau-$

จ $96^{\circ} 6 \varepsilon[-]$
$9 \varepsilon 0^{\circ}$ ObI

$288^{\circ} 8 \mathrm{ST} T-2$

$588.85 I-$

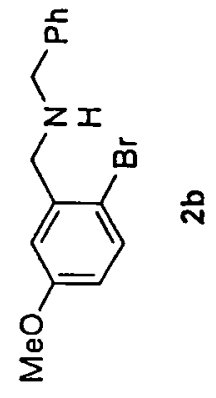

$\because \mathrm{O}$

$\ddot{u} \rightleftharpoons$ 
(C) 2003 American Chemical Society, J. Org. Chem., Kamimura jo030052h Supporting Info Page 10

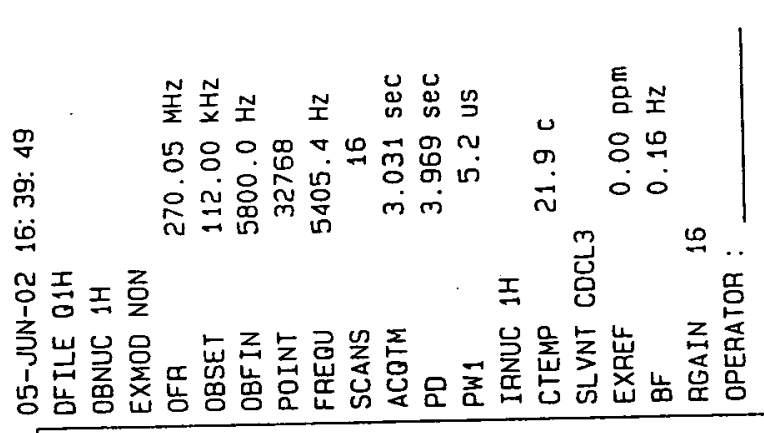

S10
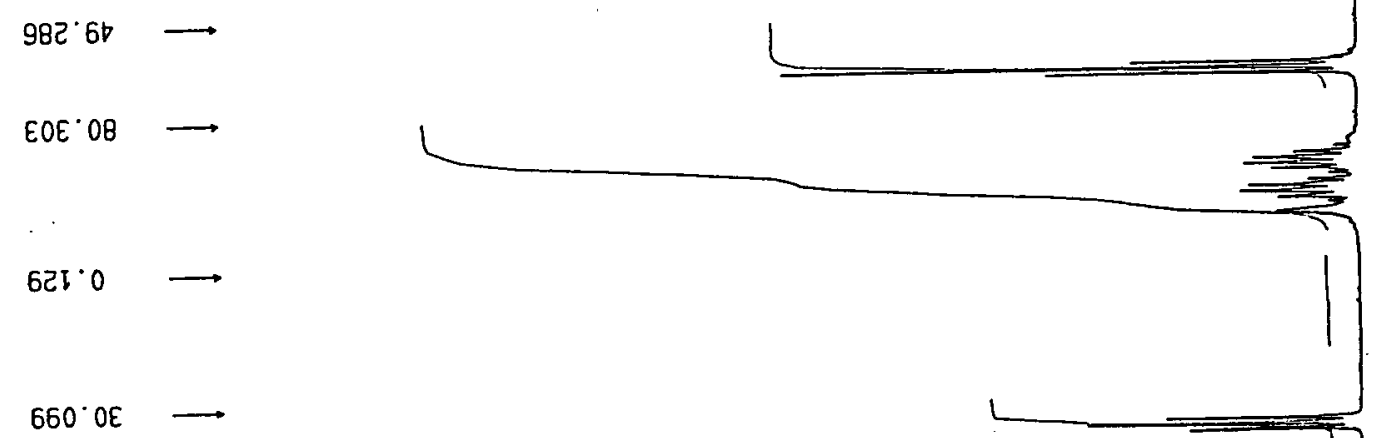

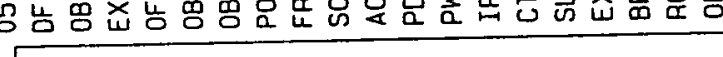

$6 \mathrm{HS}^{\circ} \mathrm{CB} \longrightarrow$

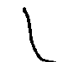

$\mathrm{OZL} \cdot \mathrm{O} \longrightarrow$

$000^{\circ} \longrightarrow \longrightarrow$

SOt'IE $\longrightarrow$

$\angle 9 L^{\circ} \angle I \quad \longrightarrow$
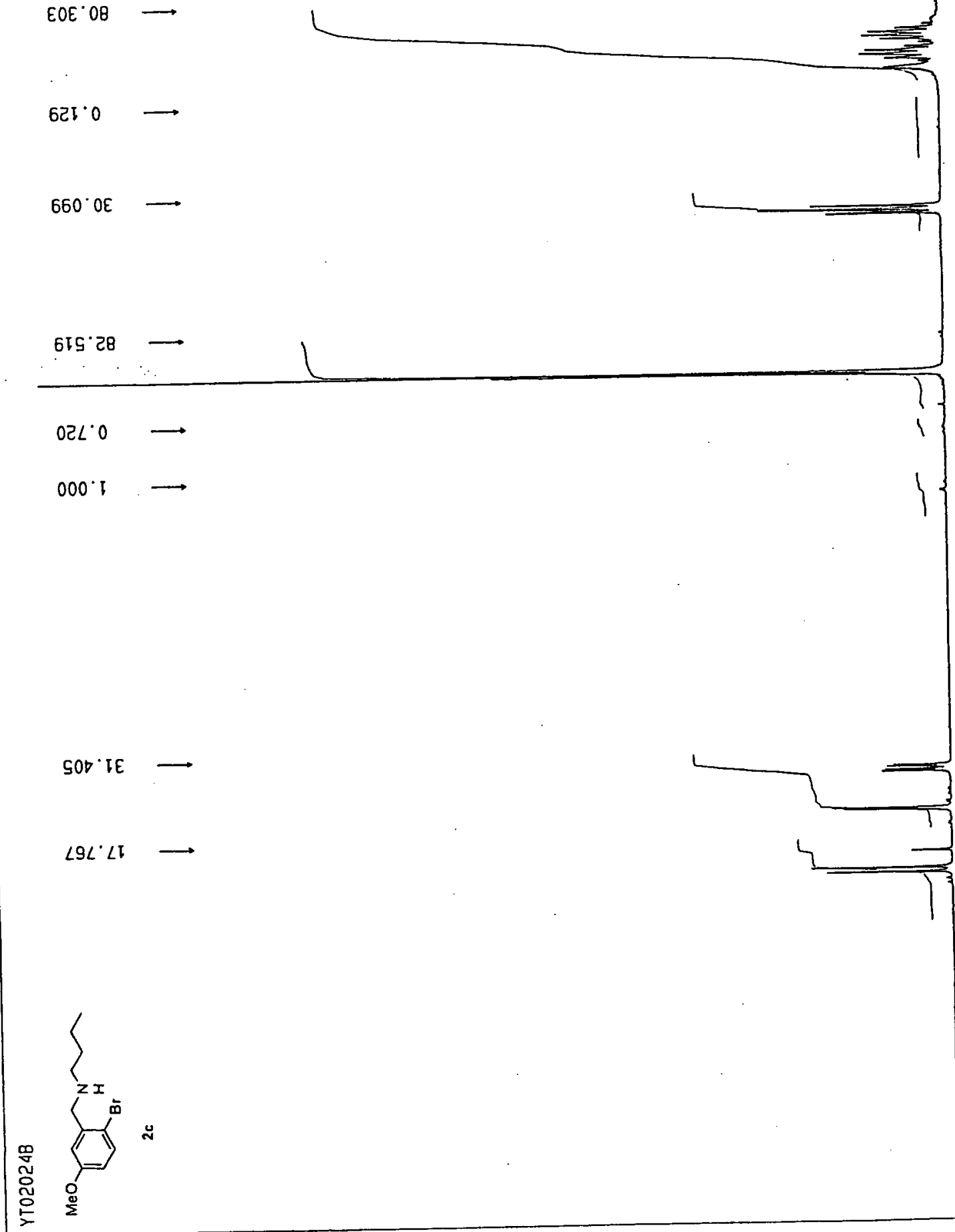
(C) 2003 American Chemical Society, J. Org. Chem., Kamimura jo030052h Supporting Info Page 11

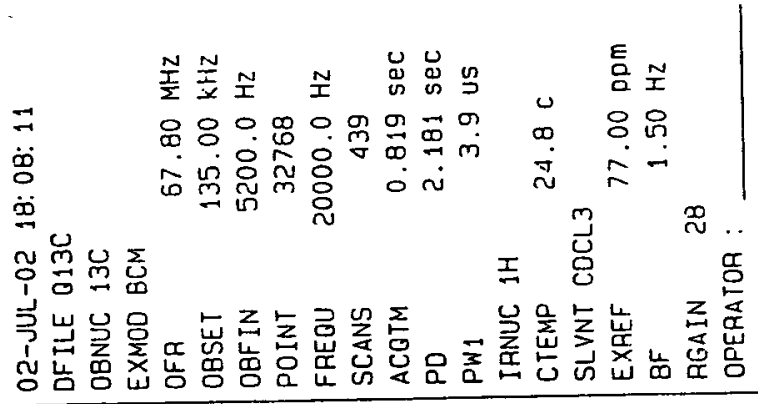

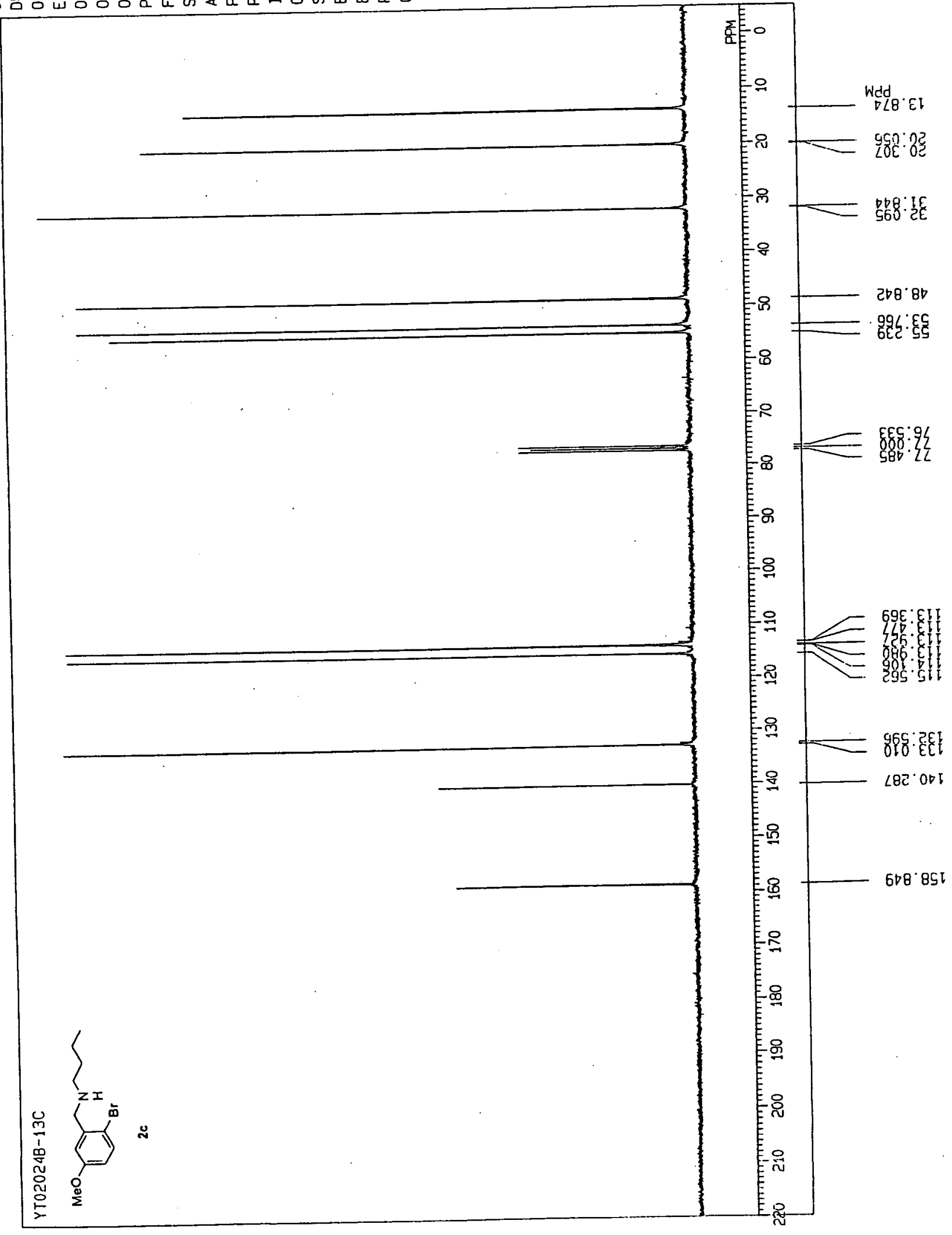


(C) 2003 American Chemical Society, J. Org. Chem., Kamimura jo030052h Supporting Info Page 12
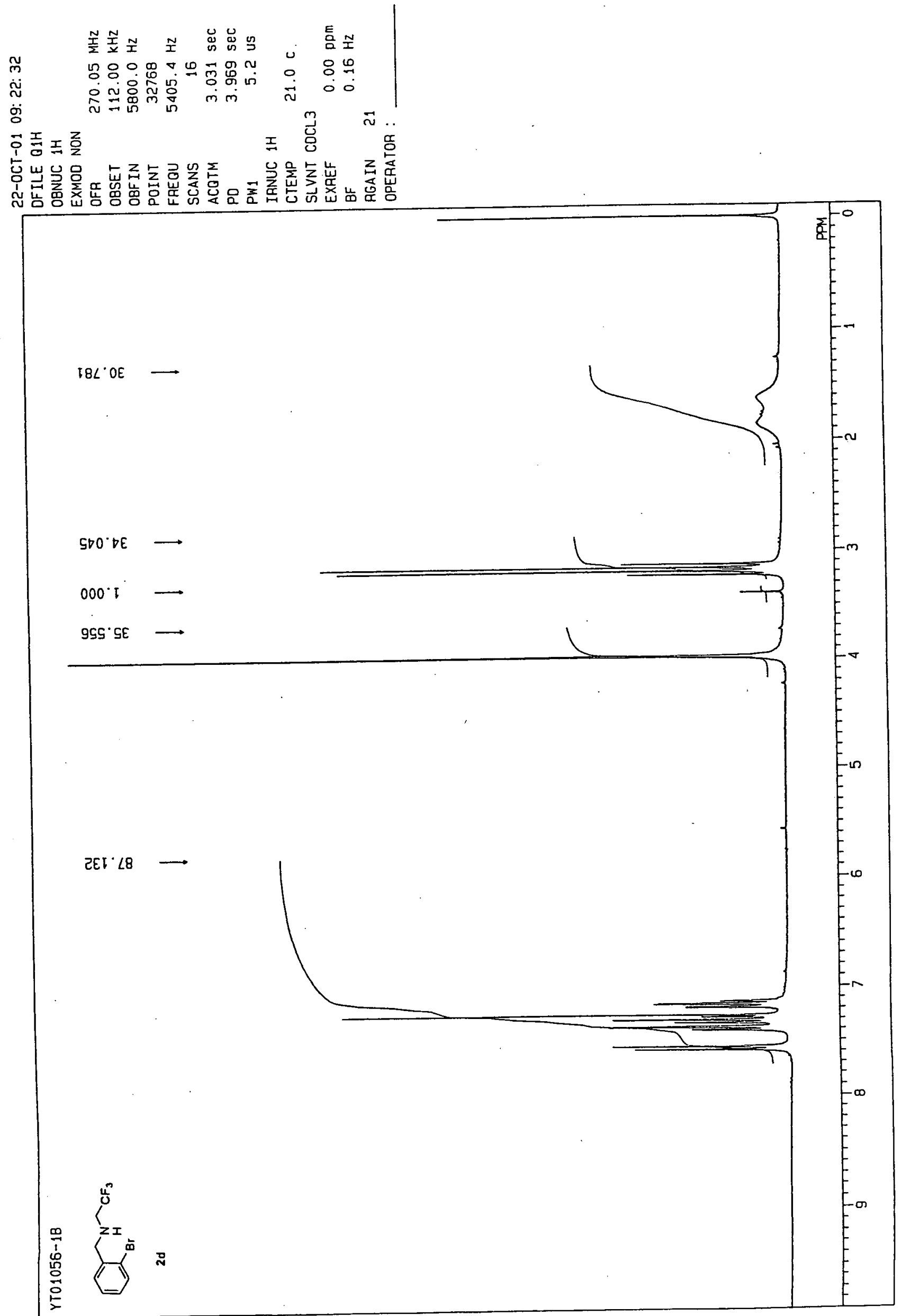
(C) 2003 American Chemical Society, J. Org. Chem., Kamimura jo030052h Supporting Info Page 13
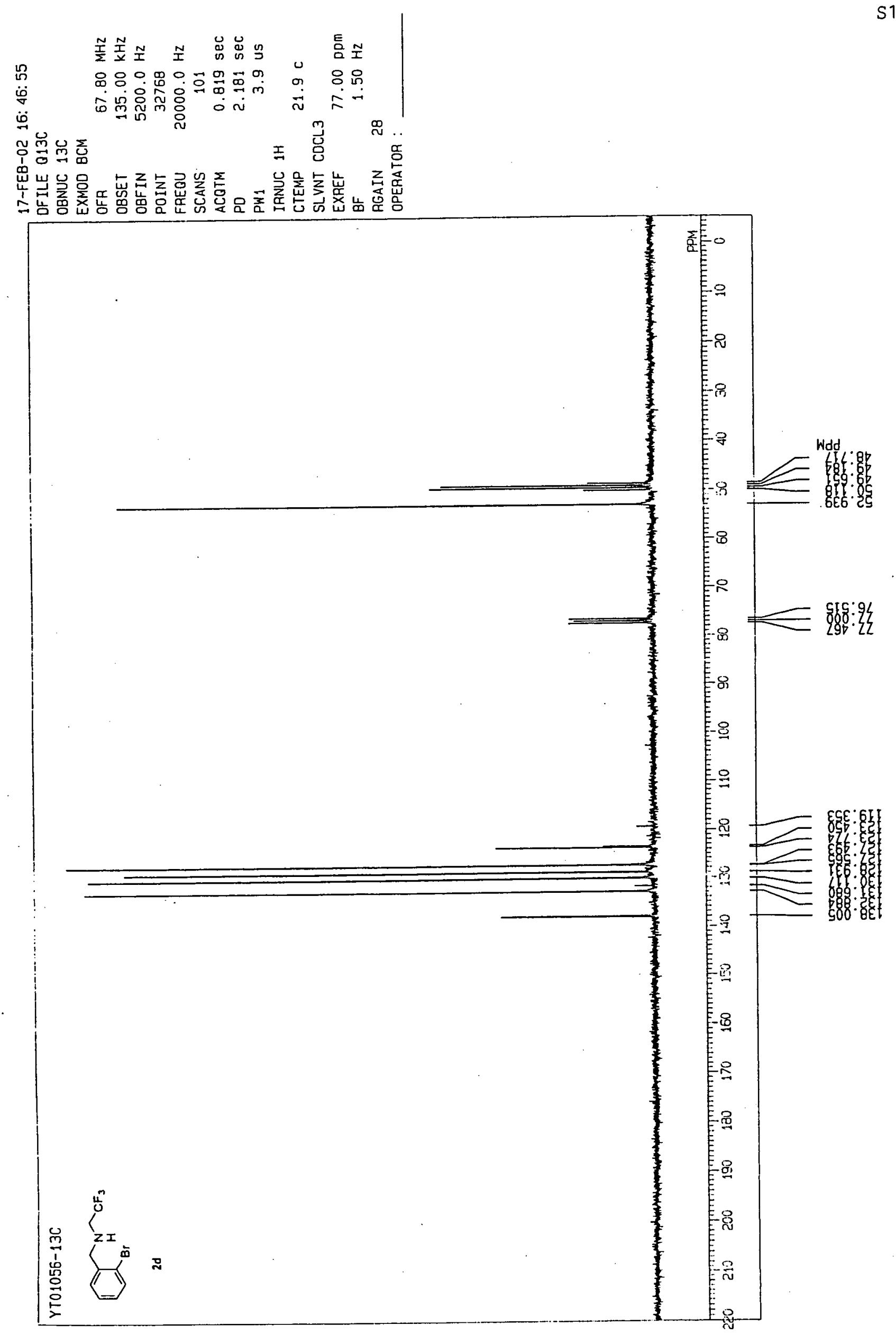
(C) 2003 American Chemical Society, J. Org. Chem., Kamimura jo030052h Supporting Info Page 14
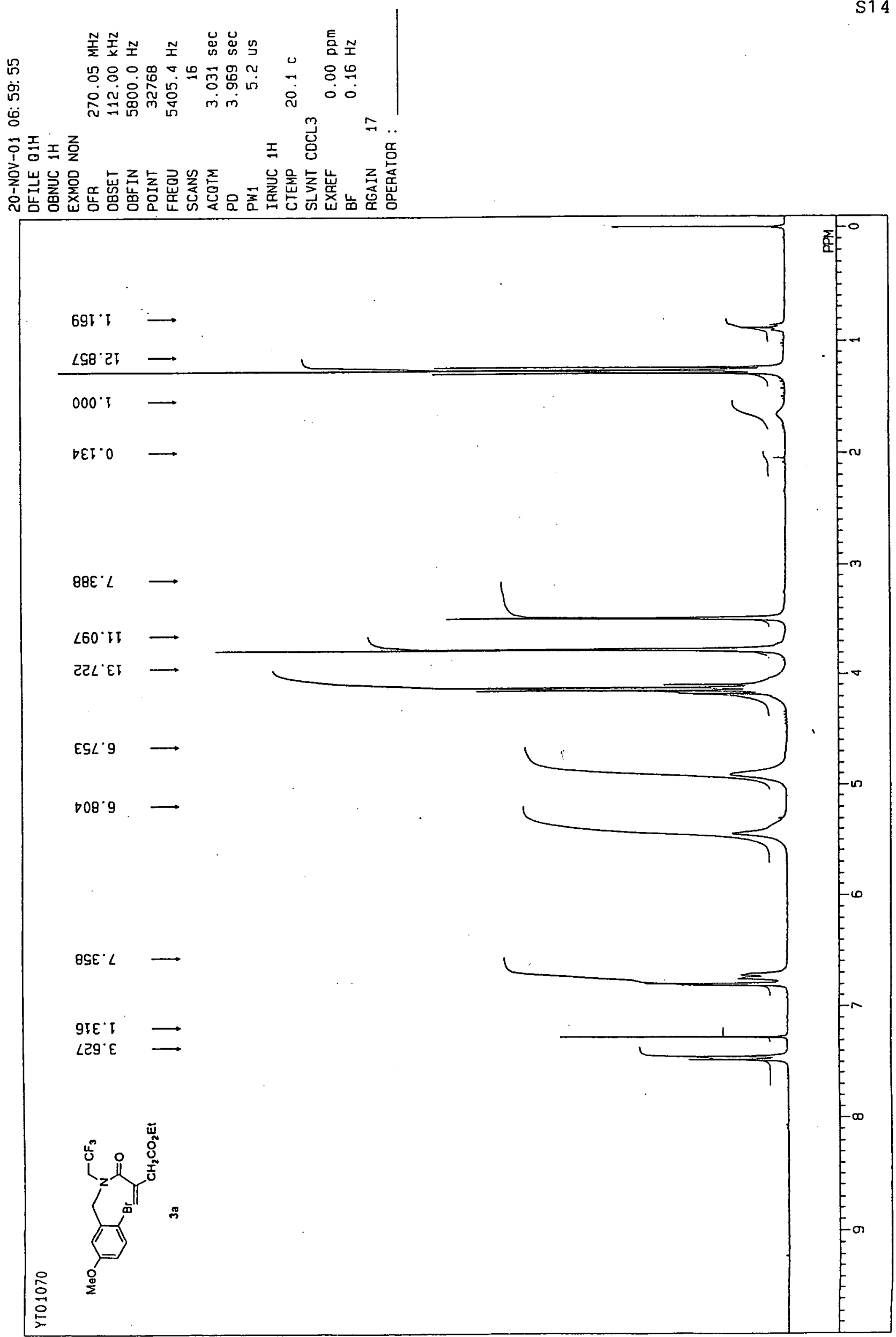
(C) 2003 American Chemical Society, J. Org. Chem., Kamimura jo030052h Supporting Info Page 15
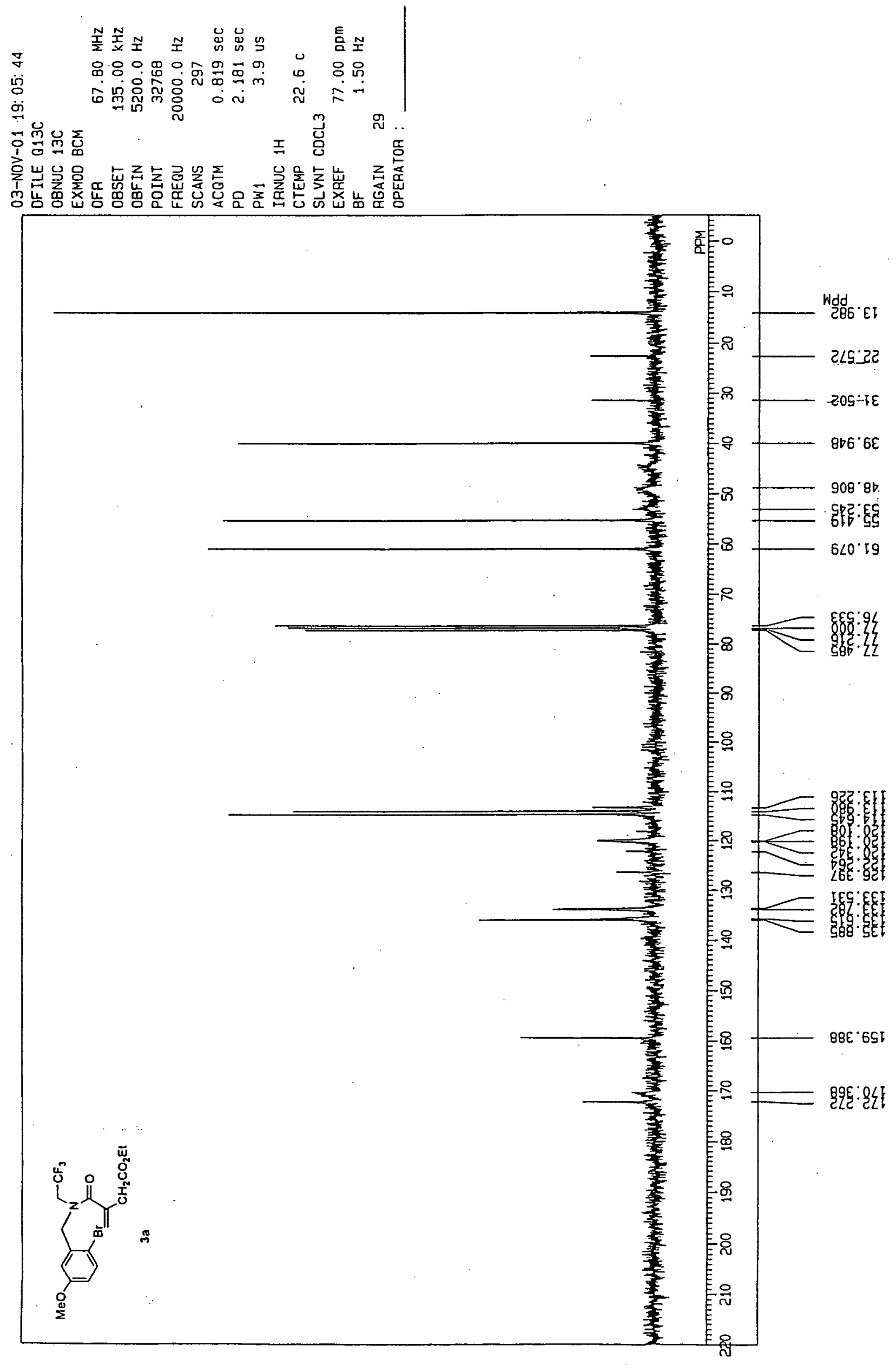
(C) 2003 American Chemical Society, J. Org. Chem., Kamimura jo030052h Supporting Info Page 16

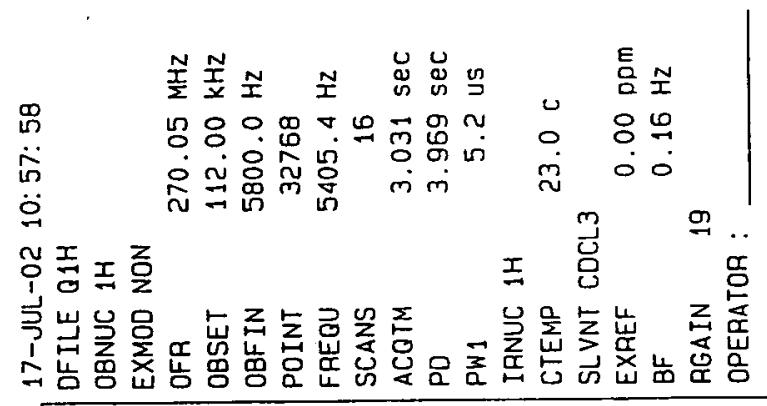

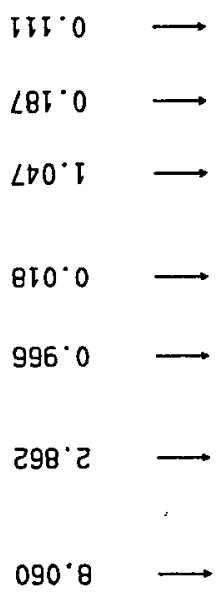

$\longrightarrow$

$\mathrm{E86} \mathrm{\circ}^{\circ} \longrightarrow$

E68. $\longrightarrow$

$000^{\circ} \mathrm{I} \longrightarrow$

$\angle E 60^{\circ} \mathrm{\longrightarrow}$

$\angle E 6^{\circ} 0 \quad \longrightarrow$

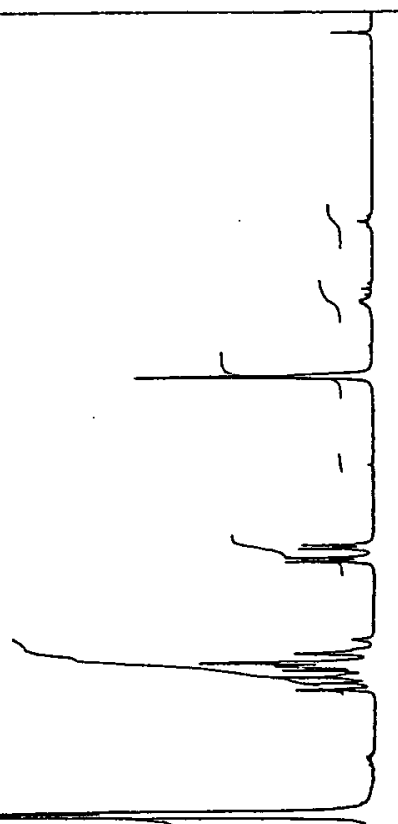

EOr's

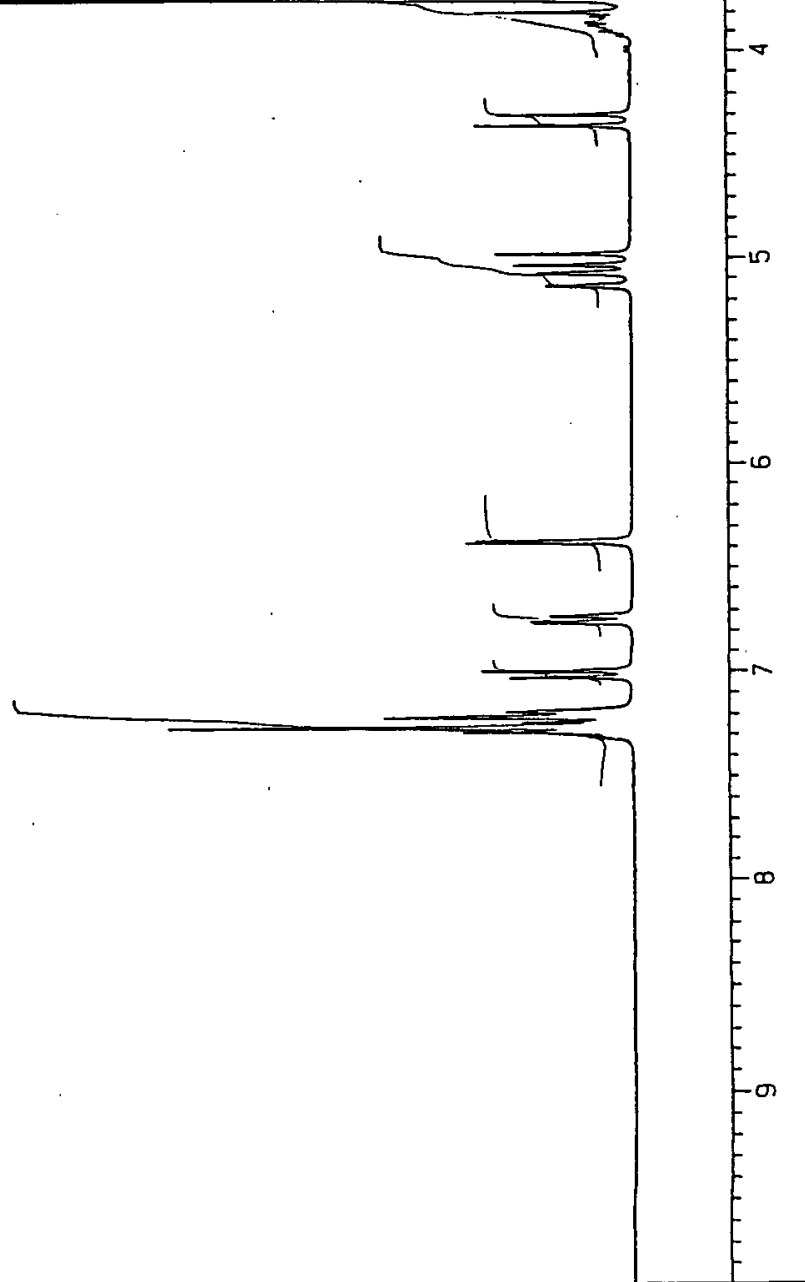


(C) 2003 American Chemical Society, J. Org. Chem., Kamimura jo030052h Supporting Info Page 17

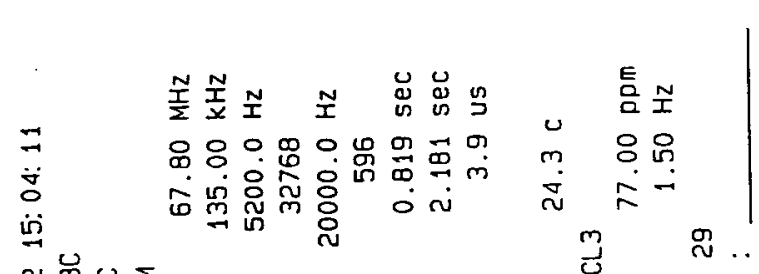

俆总兽

I

ㄸ.

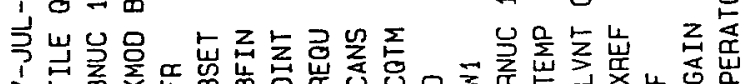

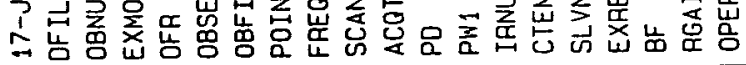

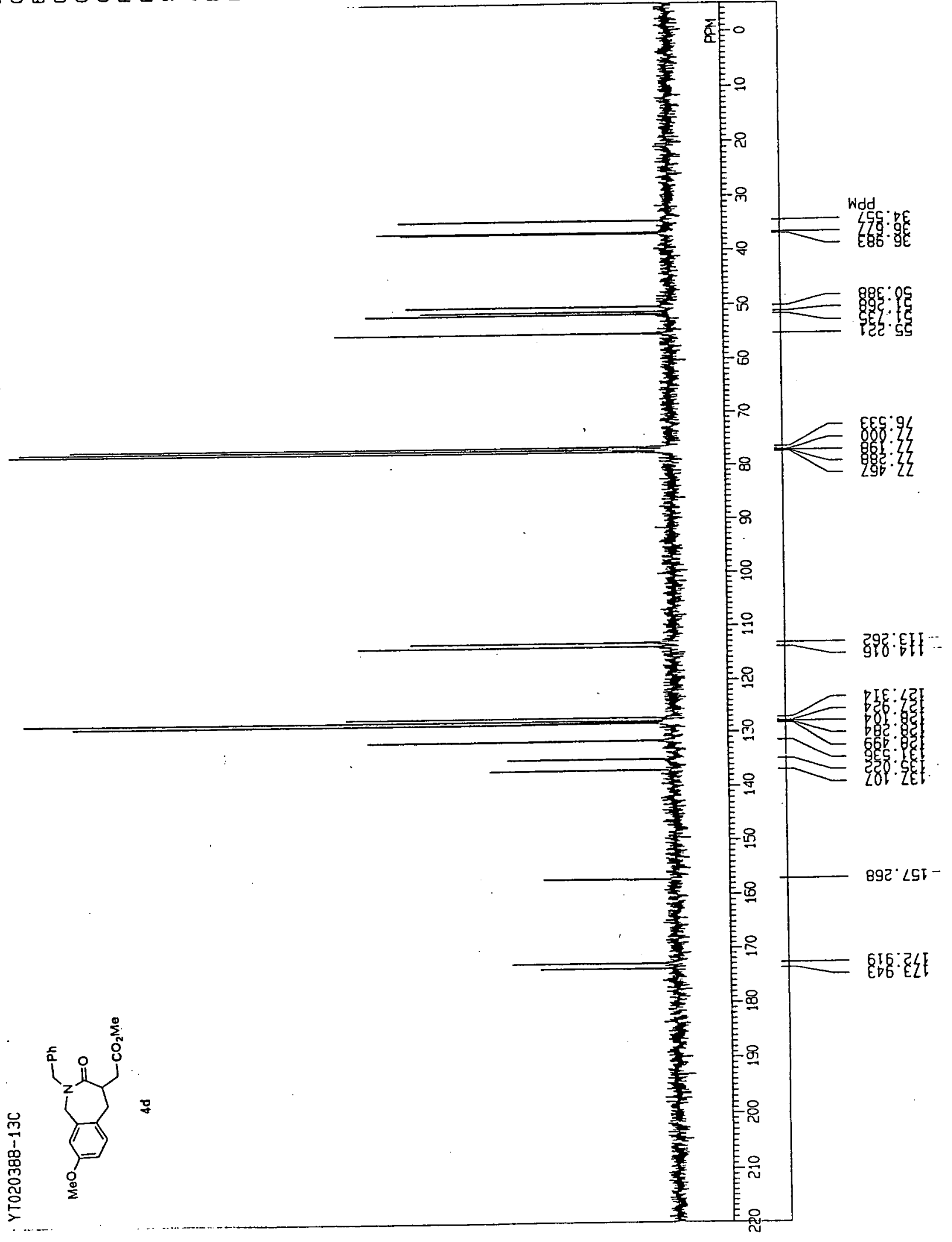



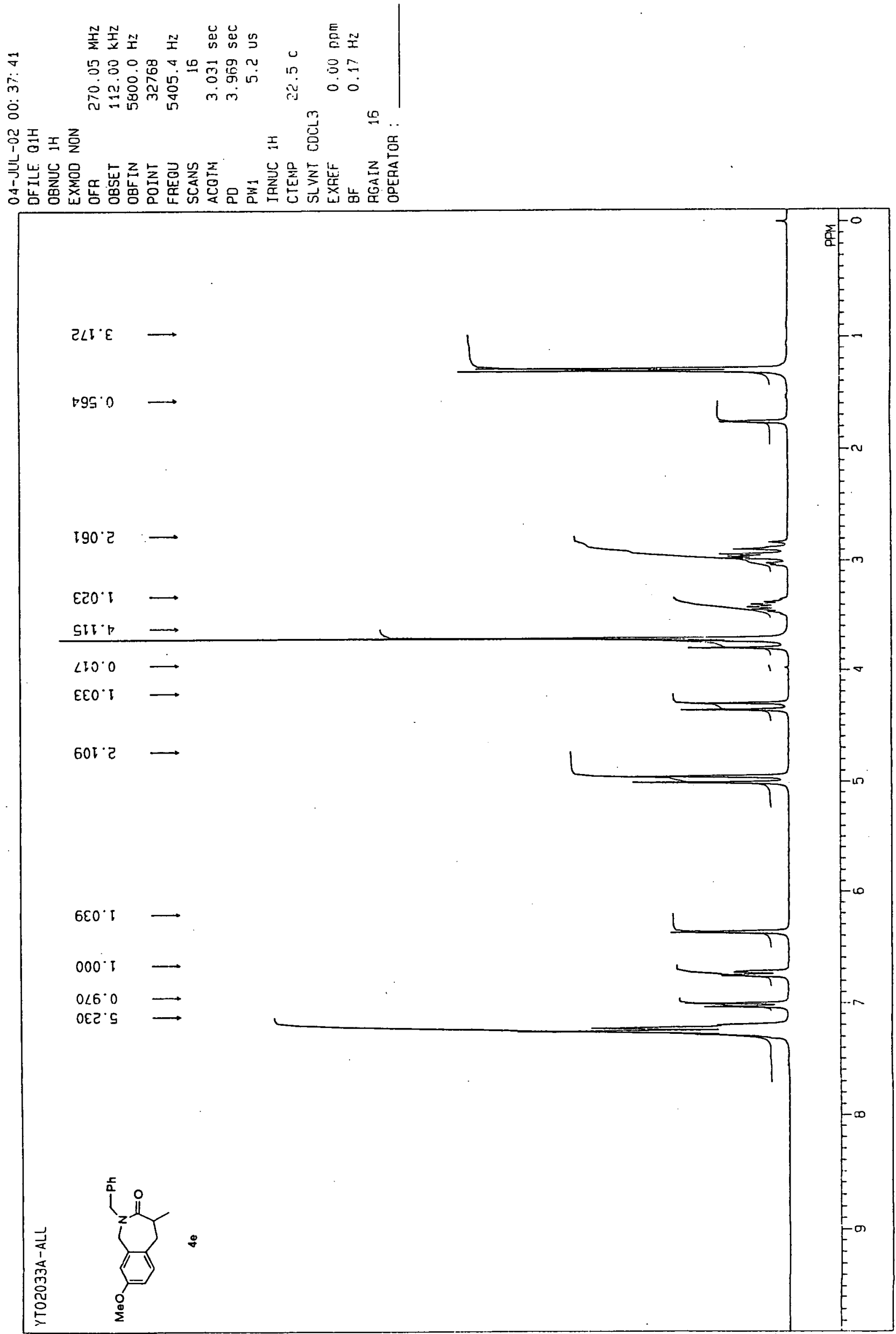
(C) 2003 American Chemical Society, J. Org. Chem., Kamimura jo030052h Supporting Info Page 19

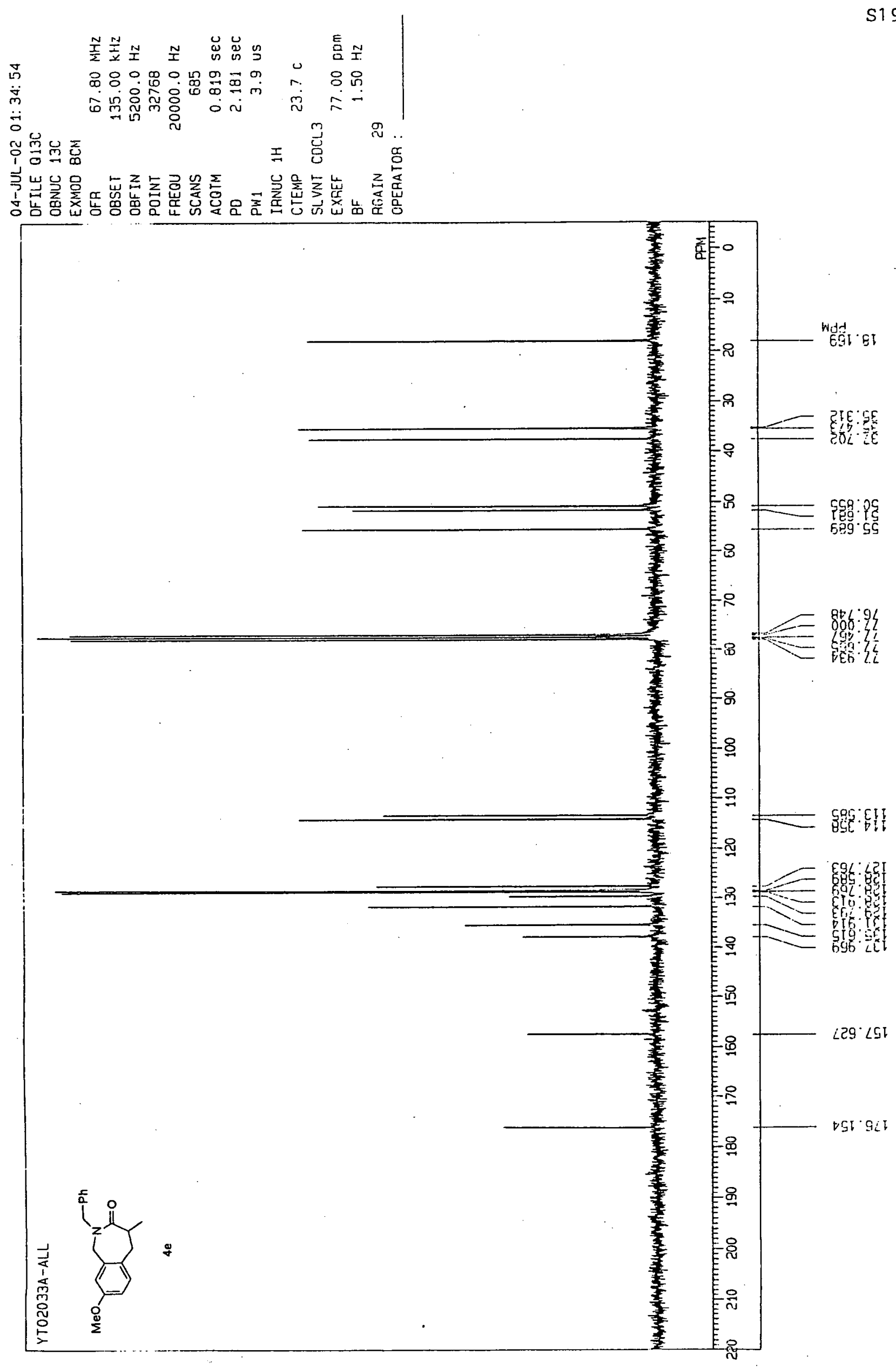




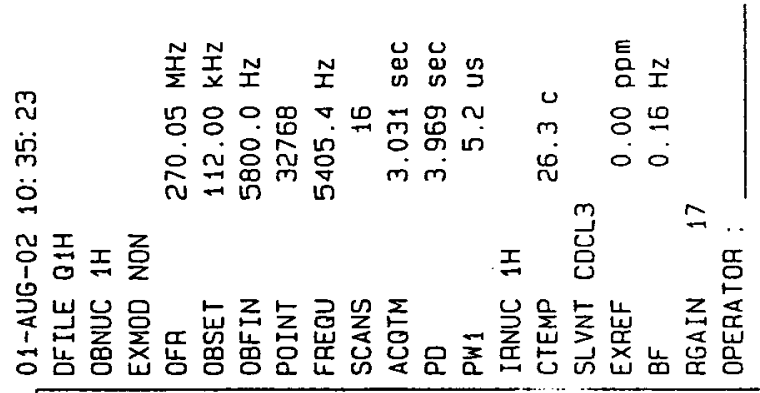

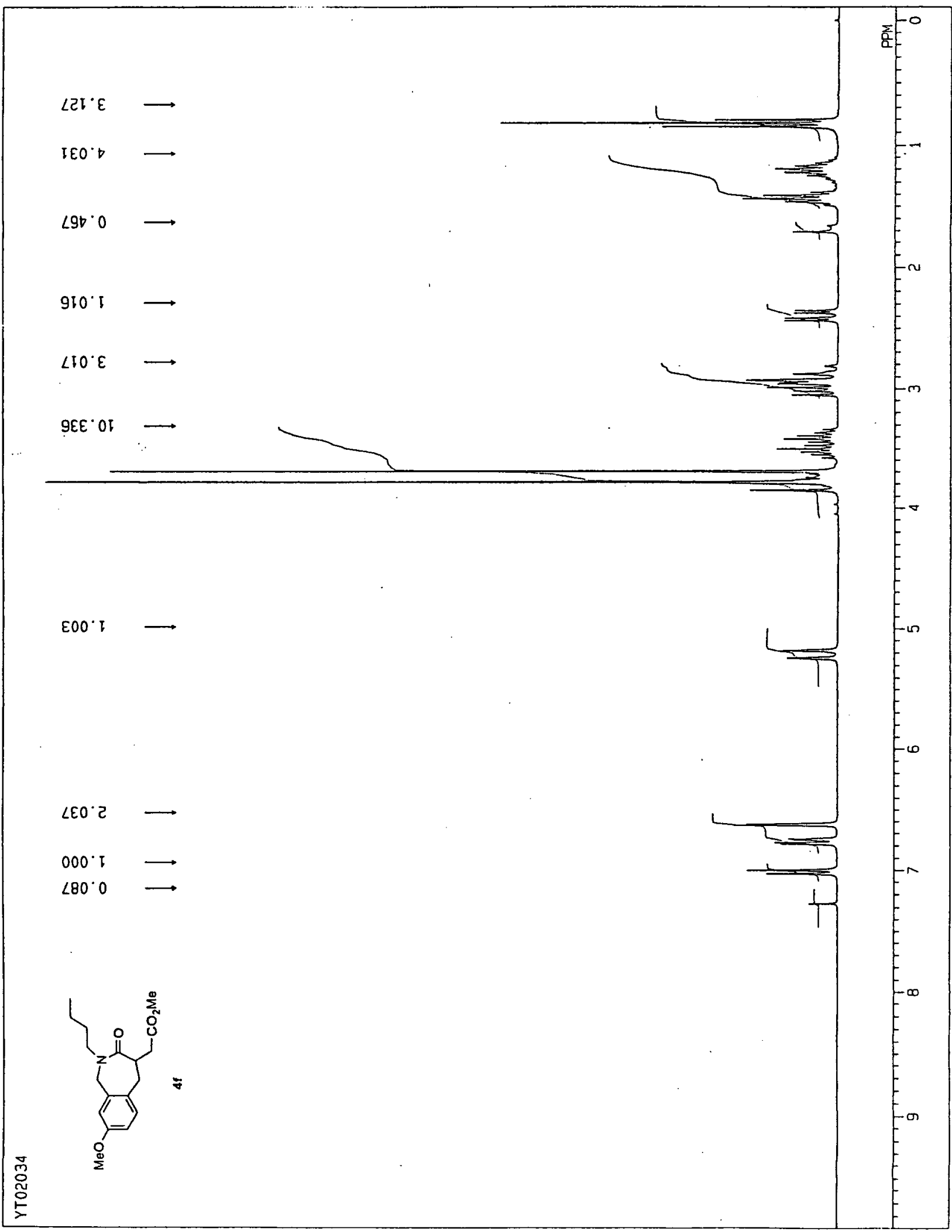



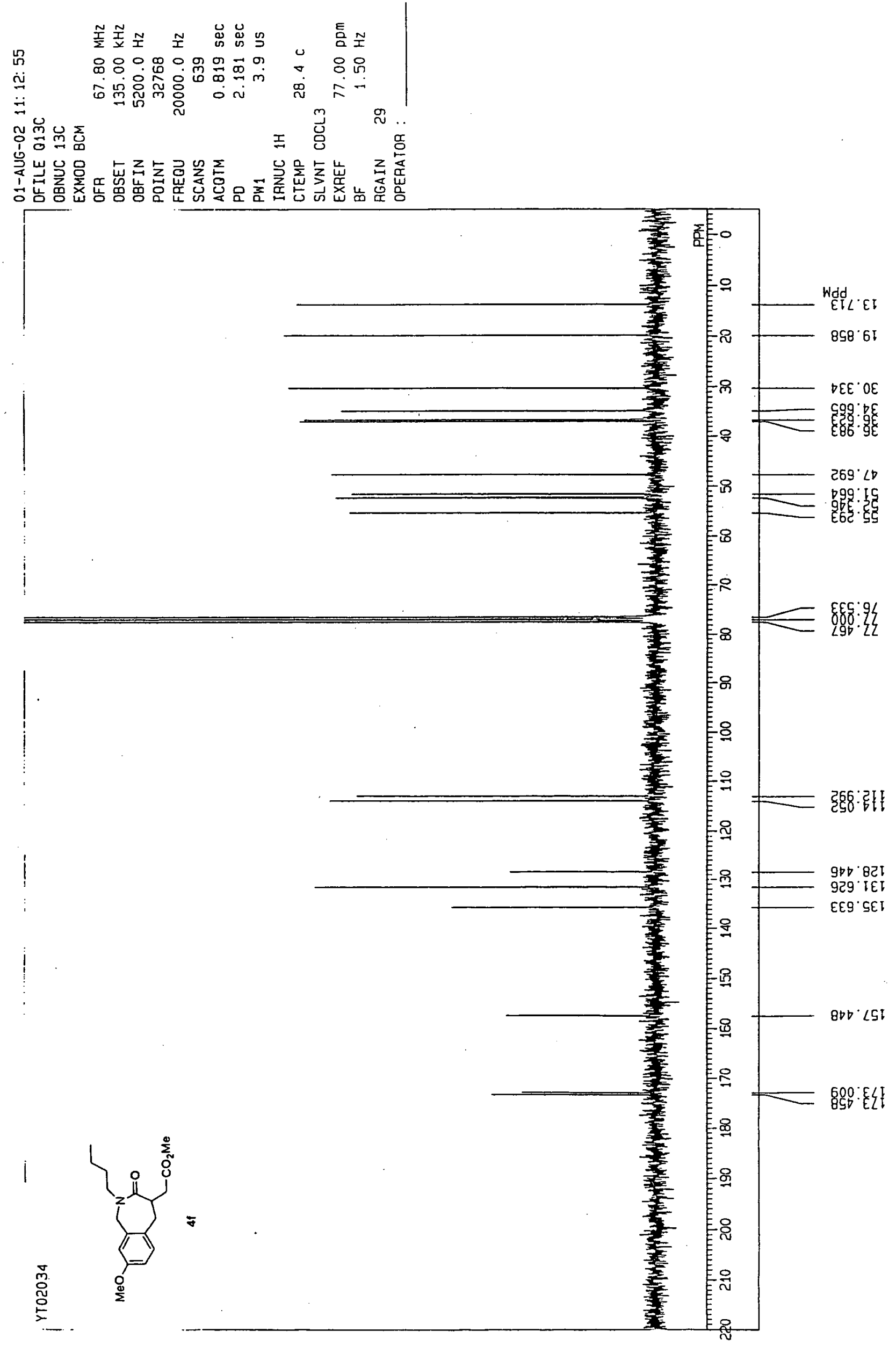
(C) 2003 American Chemical Society, J. Org. Chem., Kamimura jo030052h Supporting Info Page 22
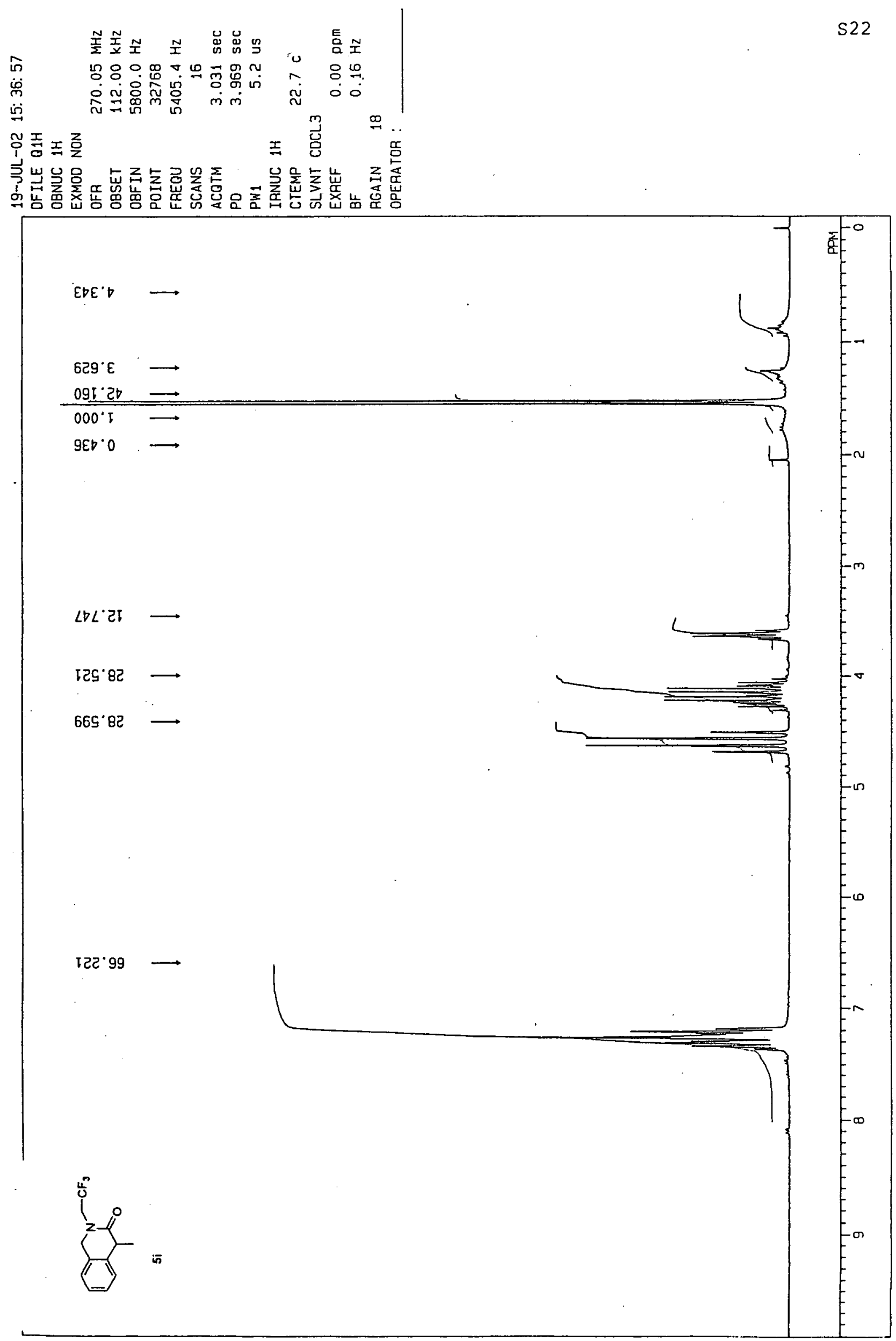
(c) 2003 American Chemical Society, J. Org. Chem., Kamimura jo030052h Supporting Info Page 23
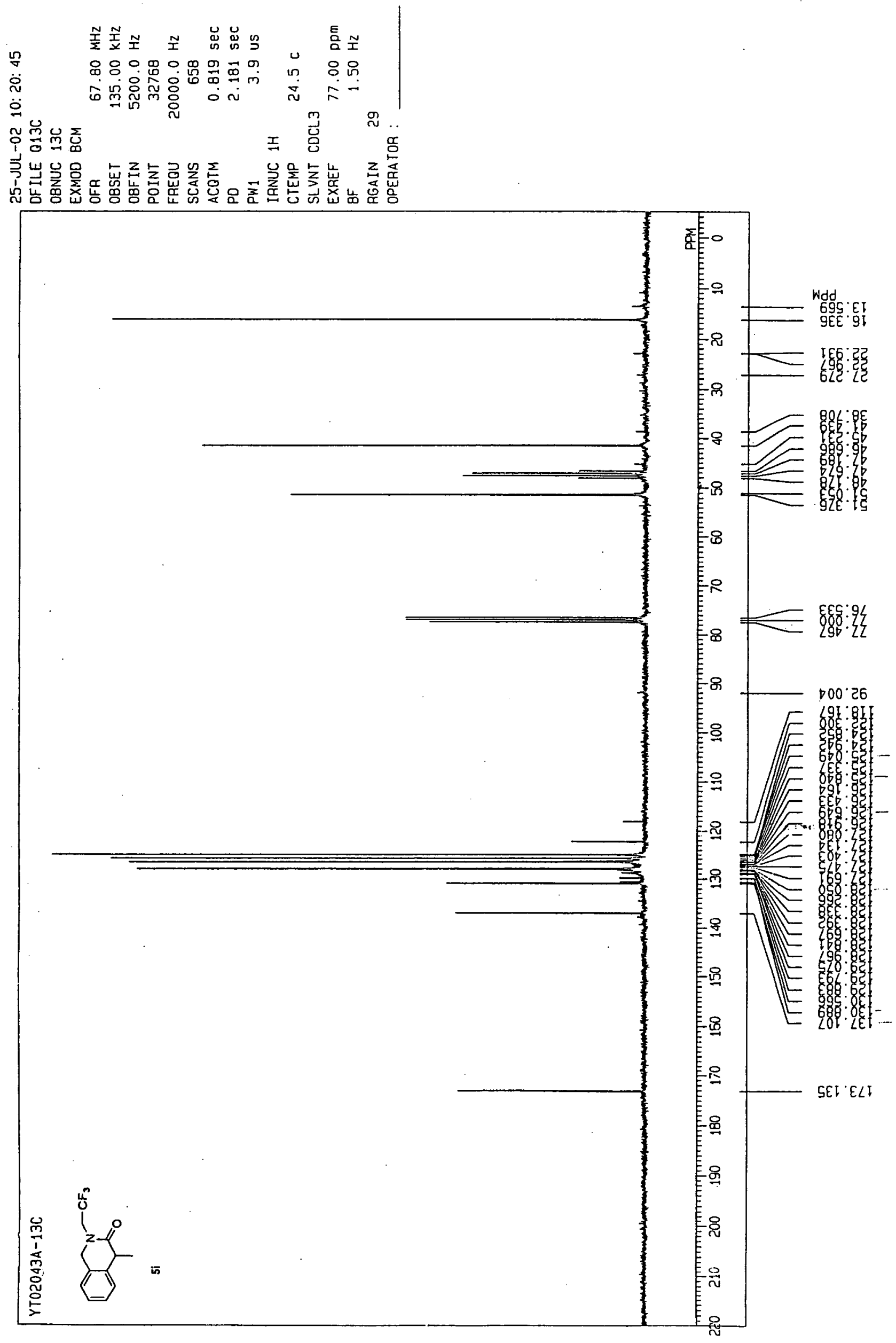
(C) 2003 American Chemical Society, J. Org. Chem., Kamimura jo030052h Supporting Info Page 24

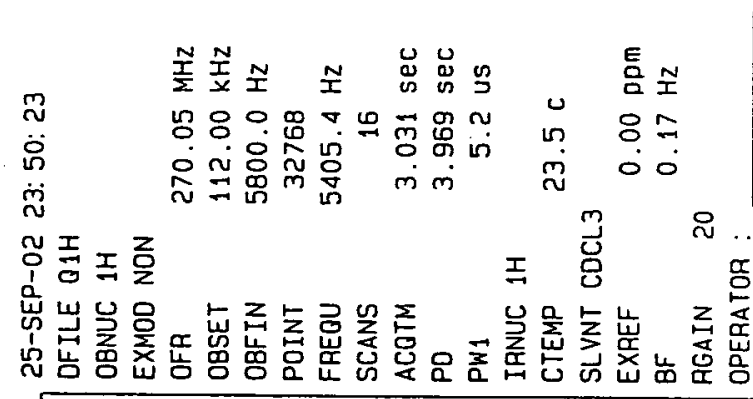

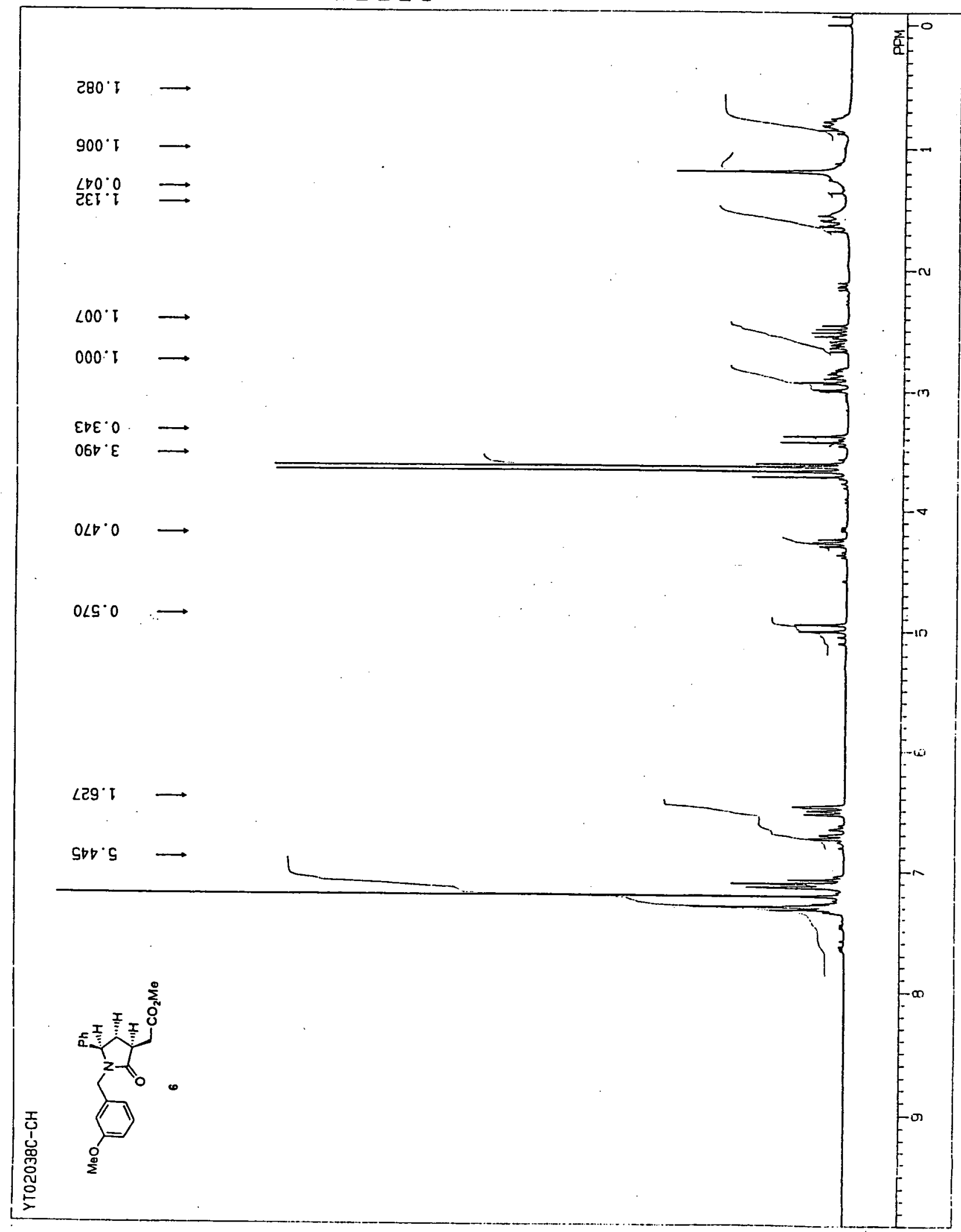



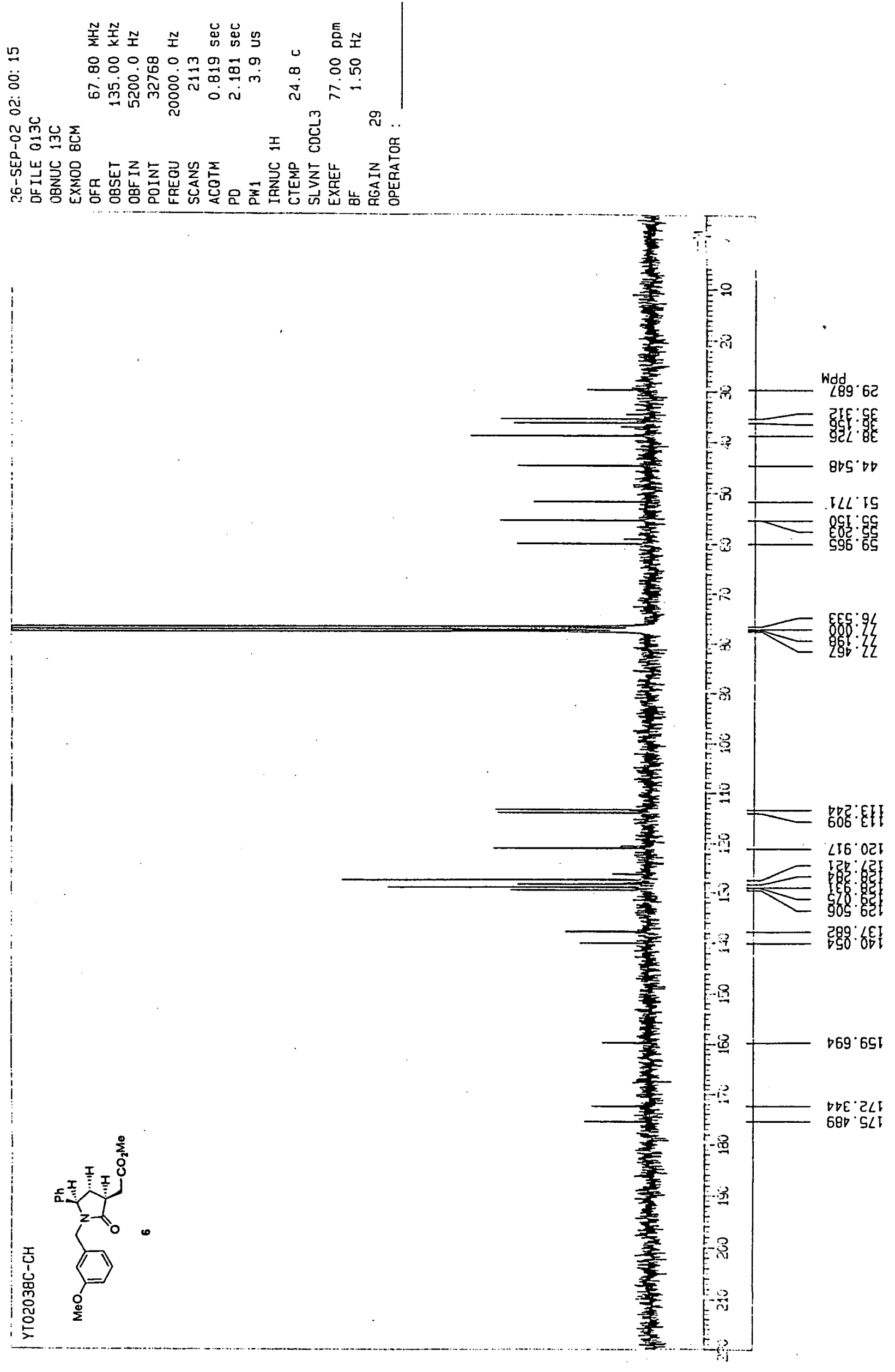